\title{
Correction to: Asymptotic properties of Lee distance
}

\author{
Nikolay I. Nikolov ${ }^{1}$ (D) Eugenia Stoimenova ${ }^{1}$
}

Published online: 20 February 2021

๑) Springer-Verlag GmbH Germany, part of Springer Nature 2021

\section{Correction to: Metrika (2019) 82:385-408 https://doi.org/10.1007/s00184-018-0687-7}

The statement of Theorem 3 (p. 397) in Nikolov and Stoimenova (2019) should be corrected to:

Theorem 3 Let $M(\theta, N)=\left\{m_{i j}(\theta, N)\right\}_{i, j=1}^{N}$ be the Marginals matrix based on Lee distance. Then

$$
m_{i j}\left(\theta_{N}, N\right) \frac{N}{\exp \left(\theta_{N} \mu+\frac{\theta_{N}^{2} \nu^{2}}{2}\right)} \underset{N \rightarrow \infty}{\longrightarrow} 1, \text { for } i, j=1,2, \ldots, N
$$

where

$$
\begin{aligned}
\mu & =\frac{N c_{N}(i, j)}{N-1}-\frac{1}{N-1}\left[\frac{N+1}{2}\right]\left[\frac{N}{2}\right], \\
v^{2} & = \begin{cases}\frac{2 N^{2}\left(c_{N}(i, j)\right)^{2}-N^{3} c_{N}(i, j)}{2(N-2)(N-1)^{2}}-\frac{N^{2}\left(N^{2}-2 N+4\right)}{48(N-1)^{2}}, & \text { for } N \text { even } \\
\frac{2 N^{2}\left(c_{N}(i, j)\right)^{2}-N\left(N^{2}-1\right) c_{N}(i, j)}{2(N-2)(N-1)^{2}}-\frac{N(N+1)(N-3)}{48(N-2)}, & \text { for } N \text { odd. }\end{cases}
\end{aligned}
$$

and $\left\{\theta_{N}\right\}_{N=1}^{\infty}$ is a sequence of real numbers such that $\lim _{N \rightarrow \infty} \theta_{N} N^{\frac{3}{2}}=a$, for some real constant a.

The original article can be found online at https://doi.org/10.1007/s00184-018-0687-7.

$\triangle$ Nikolay I. Nikolov

n.nikolov@math.bas.bg

Eugenia Stoimenova

jeni@math.bas.bg

1 Institute of Mathematics and Informatics, Bulgarian Academy of Sciences, Acad. G. Bontchev Str., Block 8, 1113 Sofia, Bulgaria 
The quantity $m_{i j}(\theta, N)$ for fixed $\theta$ in the proof $(p$. 407) of the original statement of Theorem 3 (p. 397) can be written as

$m_{i j}(\theta, N)=\frac{1}{N} \exp \left(\theta(\tilde{\mu}(N)-\mu(N))+\frac{\theta^{2}}{2}\left(\tilde{\sigma}^{2}(N)-\sigma^{2}(N)\right)\right) \frac{1+\tilde{f}(\theta \tilde{\sigma}(N), N)}{1+f(\theta \sigma(N), N)}$

where $\mu(N)=\mathbf{E}\left(D_{L}\right), \sigma^{2}(N)=\operatorname{Var}\left(D_{L}\right), \tilde{\mu}(N)=\mathbf{E}\left(\tilde{D}_{N-1}\right), \tilde{\sigma}^{2}(N)=$ $\operatorname{Var}\left(\tilde{D}_{N-1}\right)$ and $f(\theta, N)$ and $\tilde{f}(\theta, N)$ are functions such that $\lim _{N \rightarrow \infty} f(\theta, N)=0$ and $\lim _{N \rightarrow \infty} \tilde{f}(\theta, N)=0$. Thus, the original statement is equivalent to

$$
\lim _{N \rightarrow \infty} \frac{1+\tilde{f}(\theta \tilde{\sigma}(N), N)}{1+f(\theta \sigma(N), N)}=1
$$

which is not trivial to be proved and even might not hold. However, if instead of fixed $\theta$ we consider a sequence of real numbers $\left\{\theta_{N}\right\}_{N=1}^{\infty}$ such that $\lim _{N \rightarrow \infty} \theta_{N} N^{\frac{3}{2}}=a$, for some real constant $a$, then

$$
\lim _{N \rightarrow \infty} \theta_{N} \sigma(N)=c, \quad \lim _{N \rightarrow \infty} \theta_{N} \tilde{\sigma}(N)=\tilde{c}
$$

where $c$ and $\tilde{c}$ are some real constants, and $\lim _{N \rightarrow \infty} \frac{1+\tilde{f}\left(\theta_{N} \tilde{\sigma}(N), N\right)}{1+f\left(\theta_{N} \sigma(N), N\right)}=1$. This completes the proof of the corrected formulation.

Furthermore, since the theorem holds for a sequence of real numbers $\left\{\theta_{N}\right\}_{N=1}^{\infty}$, it could be used to approximate the Marginals matrix $M(\theta, N)$ only when the value of $\theta$ is close to 0 . However, our experience from simulation studies indicates that the original statement of Theorem 3 ( $p$. 397) might be true and the approximation could be applied even for large values of $\theta$. As an illustration, see the example in Section 4.2 (pp. 400-402).

Acknowledgements The authors would like to thank Prof. Mladen Savov for his careful review and constructive comments in relation to this work.

\section{Reference}

Nikolov N, Stoimenova J (2019) Asymptotic properties of Lee distance. Metrika 82:385-408

Publisher's Note Springer Nature remains neutral with regard to jurisdictional claims in published maps and institutional affiliations. 